\title{
Sex differences in umbilical artery Doppler indices: a longitudinal study
}

Christian Widnes ${ }^{1,2^{*}}$ (D), Kari Flo ${ }^{1,2}$, Tom Wilsgaard ${ }^{3}$, Torvid Kiserud ${ }^{4,5}$ and Ganesh Acharya ${ }^{1,2,6}$

\begin{abstract}
Background: Sexual dimorphism in placental size and function has been described. Whether this influences the clinically important umbilical artery (UA) waveform remains controversial, although a few cross-sectional studies have shown sex differences in UA pulsatility index (PI). Therefore, we tested whether fetal sex influences the UA Doppler indices during the entire second half of pregnancy and aimed to establish sex-specific reference ranges for UA Doppler indices if needed.

Methods: Our main objective was to investigate gestational age-associated changes in UA Doppler indices during the second half of pregnancy and compare the values between male and female fetuses. This was a prospective longitudinal study in women with singleton low-risk pregnancies during 19-40 weeks of gestation. UA Doppler indices were serially obtained at a 4-weekly interval from a free loop of the umbilical cord using color-directed pulsed-wave Doppler ultrasonography. Sex-specific reference intervals were calculated for the fetal heart rate (HR), UA PI, resistance index (RI), and systolic/diastolic ratio (S/D) using multilevel modeling.

Results: Complete data from 294 pregnancies (a total of 1261 observations from 152 male and 142 female fetuses) were available for statistical analysis, and sex-specific reference ranges for the UA Doppler indices and fetal HR were established for the last half of pregnancy. UA Doppler indices were significantly associated with gestational age ( $P$ $<0.0001)$ and fetal HR $(P<0.0001)$. Female fetuses had $2-8 \%$ higher values for UA Doppler indices than male fetuses during gestational weeks $20^{+0}-36^{+6}(P<0.05)$, but not later. Female fetuses had higher HR from gestational week $26^{+0}$ until term $(P<0.05)$.
\end{abstract}

Conclusions: We have determined gestational age-dependent sex differences in UA Doppler indices and fetal HR during the second half of pregnancy, and correspondingly established new sex-specific reference ranges intended for refining diagnostics and monitoring individual pregnancies.

Keywords: Fetal Doppler, Obstetric ultrasound, Placental blood flow, Sex differences, Umbilical artery, Reference ranges

\section{Background}

The importance of conducting longitudinal studies and analyzing data accounting for biological differences related to sex has been highlighted a decade ago [1]. Previous studies have shown sex-specific differences in fetal development regarding growth and adaption to intrauterine environment [2]. Male sex is an independent risk

\footnotetext{
* Correspondence: Christian.Widnes@unn.no

${ }^{1}$ Women's Health and Perinatology Research Group, Department of Clinical Medicine, Faculty of Health Sciences, UiT-The Arctic University of Norway, Tromso, Norway

${ }^{2}$ Department of Obstetrics and Gynecology, University Hospital of North Norway, Sykehusveien 38, PO Box 24, N-9038 Tromso, Norway

Full list of author information is available at the end of the article
}

factor for unfavorable perinatal outcomes including fetal distress during labor $[3,4]$, premature birth $[5,6]$, adverse neonatal outcome [7], and early neonatal death [2]. This has been referred to as "the male disadvantage" [8] and the female neonatal survival advantage is well recognized [9]. However, the total mortality from conception to birth is greater among female fetuses [10]. The human placenta demonstrates sex-related differences at both structural and functional levels [11, 12]. Both birth weight and placental weight [13] are higher for males compared with females. Sexual dimorphism in the regulation and expression of genes, and signaling pathways [14-17], generate differences in placental function and

(c) The Author(s). 2018 Open Access This article is distributed under the terms of the Creative Commons Attribution 4.0 International License (http://creativecommons.org/licenses/by/4.0/), which permits unrestricted use, distribution, and 
intrauterine environment that may lead to sex differences in health status later in life $[12,18]$.

Antenatal growth charts show significant differences in biometrics between male and female fetuses [19]. When using two-dimensional ultrasonography to assess fetal growth, these sex-specific growth charts perform better in identifying small for gestational age (SGA) fetuses [20] at increased risk of fetal demise [21].

Umbilical artery (UA) Doppler indices, i.e., pulsatility index (PI), resistance index (RI), and systolic/diastolic ratio (S/D) calculated from blood flow velocities, are used as an important clinical tool for evaluating fetal wellbeing in high-risk pregnancies and to predict outcome of growth restricted fetuses [22]. Their use in high-risk pregnancies has the potential to reduce obstetric interventions and perinatal deaths [23]. The increased UA PI is a marker of raised placental vascular impedance associated with microvascular lesions [24] and correspondingly reduced placental function. Longitudinal reference ranges for UA Doppler indices calculated from both cross-sectional and serial measurements have previously been published $[25,26]$. However, these studies do not take into account possible sex differences.

Doppler ultrasonographic studies exploring fetal sex differences in the ductus venosus during the first trimester have shown antagonistic results [27-29]. Another study performed just prior to active labor, in term pregnancies, demonstrated no differences in UA PI, but statistically significant lower values for the middle cerebral artery (MCA) PI, MCA peak systolic velocity (PSV) and normalized umbilical venous blood flow ( $\mathrm{Quv}_{\mathrm{uv}}$ in male compared with female fetuses [30], but these differences did not translate into differences in perinatal outcome. One recent study of the feto-placental circulation and cardiac function during 28-34 gestational weeks showed higher preload and lower afterload (significantly lower UA PI) in male fetuses [31]. In a cross-sectional study investigating maternal hemodynamics and placental circulation, we recently demonstrated significantly lower UA PI in male compared with that in female fetuses at $22^{+0}-24^{+0}$ weeks of gestation [32].

Based on such observations, the main objective of our present study was to assess the effect of fetal sex on UA Doppler indices during the entire second half of pregnancy and correspondingly establish sex-specific longitudinal reference ranges for clinical use.

\section{Methods}

In this study, we used data from a total of 306 healthy pregnant women with uncomplicated singleton pregnancy participating in three prospective longitudinal observational studies that included investigation of fetoplacental hemodynamics. The women, all $>18$ years old, were recruited at the time of routine ultrasound screening at 17-20 weeks of gestation at the University Hospital of North Norway. The gestational age was based on the biometry of fetal head performed during this scan. Women with singleton pregnancy with no history of any systemic diseases that may affect the course and outcome of pregnancy were included. A history of preeclampsia, intrauterine growth retardation (IUGR), gestational diabetes or preterm labor before 34 weeks in previous pregnancy, multiple pregnancy, maternal smoking, or presence of any chromosomal or major structural fetal anomaly in the current pregnancy were reasons for exclusion. The study protocols were approved by the Regional Committee for Medical and Health Research Ethics -North Norway (REK Nord 74/2001, 52/2005, and $105 / 2008)$, and informed written consent was obtained from each participant.

For Doppler ultrasonography, an Acuson Sequoia 512 ultrasound system with a $6-\mathrm{MHz}$ curvilinear transducer (Mountain View, CA, USA) or a Vivid 7 Dimension ultrasound system equipped with a $4 \mathrm{MS}$ sector transducer with frequencies of 1.5-4.3 $\mathrm{MHz}$ (GE Vingmed Ultrasound AS, Horten, Norway) was used. Four experienced clinicians performed the examinations at approximately 4-weekly intervals. In two of the studies all measurements were performed by two single operators, and in the third study three different operators did the examinations. The sex of the fetus was neither acknowledged nor recorded prenatally during ultrasonography. Each participant was examined 3-6 times by the same clinician, starting from 19 to 22 gestational weeks until delivery. The estimated fetal weight (EFW) was computed at each visit from measurements of the biparietal diameter (BPD), abdominal circumference (AC), and femur length (FL) based on the Hadlock 2 formula [33]. Blood flow velocity waveforms of the UA were obtained from the free-floating loop of the umbilical cord using pulsed-wave Doppler optimizing the insonation with simultaneous use of color Doppler. The angle of insonation was always kept $<15$ degrees, and angle correction was used if the angle was not zero. To ensure Doppler recording of the spatial maximum blood velocity, an expanded sample gate of 5-12 mm was used depending on gestational age. The high-pass filter was set at low. The blood flow velocities (i.e., PSV, end-diastolic velocity (EDV), and time-averaged maximum velocity (TAMXV)) , and fetal heart rate (HR) were measured online using the maximum velocity envelope recorded over the cardiac cycle. An average of three consecutive cycles were used for analysis. The ALARA (as low as reasonably achievable) principle [34] was employed. At all times during the ultrasonographic examination the mechanical and thermal indices were kept below 1.9 and 1.5, respectively. We recorded the UA blood flow successfully in 1243 out of 1261 (98.57\%) observations. The UA 
Doppler indices were calculated from the recorded velocities as follows: PI $=(\mathrm{PSV}-\mathrm{EDV}) / \mathrm{TAMXV}$ [35], RI $=(\mathrm{PSV}-\mathrm{EDV}) / \mathrm{PSV}$ [36], and S/D ratio $=\mathrm{PSV} / \mathrm{EDV}$ [37].

The reproducibility of the Doppler parameters studied, expressed by the intra-observer coefficient of variation $(\mathrm{CV})$ and the inter-observer $\mathrm{CV}$, has previously been assessed and reported [26, 38, 39].

All women had a regular antenatal follow-up according to local guidelines. Following delivery, the course and outcome of pregnancy, including any maternal or fetal complications, gestation at delivery, mode of delivery, birth weight, placental weight, neonatal sex, Apgar scores, umbilical cord blood acid-base status, and neonatal outcome was obtained from the electronic medical records. On the second day post-partum, a pediatrician routinely examined all neonates prior to discharge.

Statistical Analysis Software version 9.3 (SAS institute Inc., Cary, NC, USA) was used for statistical analysis. Logarithmic or power transformations were performed on all numerical variables that were not normally distributed, in order to best meet the criteria of normal distribution. The best transformation for each variable was determined using the Box-Cox regression. Fractional polynominals were used to achieve best-fitting curves in relation to gestational age for each variable, accommodating for nonlinear associations. We used multilevel modeling to construct gestational age-specific reference percentiles from each fitted model according to Royston and Altman [40]. The comparison between groups was done using independent samples $t$ test for continuous variables, while the chi-square test was used for categorical variables. The comparison of UA Doppler indices between male and female fetuses was performed for each gestational week after having checked and adjusted for possible confounding factors (fetal HR, EFW, and placental weight) by including a cross-product term between sex and gestational age in the aforementioned multilevel models. The level of statistical significance was set at a two-tailed $p$ value of $<0.05$.

\section{Results}

From a total study population of 306 women, 12 were excluded because they had $<3$ observations, leaving complete data from 294 pregnancies available for statistical analysis. There were 152 male and 142 female fetuses. The baseline characteristics of the study population, including pregnancy and neonatal outcomes, are presented in Table 1 . We observed no statistically significant differences between the two groups in any of the listed baseline variables.

There were 650 and 611 observations for male and female fetuses, respectively. The UA Doppler indices (PI, $\mathrm{RI}$, and S/D ratio) and the fetal HR were significantly associated with gestational age $(P<0.0001)$, and there was
Table 1 Baseline characteristics of the study population and pregnancy outcomes

\begin{tabular}{|c|c|c|c|}
\hline & $\begin{array}{l}\text { Female } \\
(n=142)\end{array}$ & $\begin{array}{l}\text { Male } \\
(n=152)\end{array}$ & $P$ value \\
\hline \multicolumn{4}{|l|}{ Maternal } \\
\hline Age (years) & 29 (range 20-43) & 30 (range 18-40) & 0.646 \\
\hline $\begin{array}{l}\text { Body mass index } \\
\text { at booking }\left(\mathrm{kg} / \mathrm{m}^{2}\right)\end{array}$ & $24.85 \pm 4.00$ & $24.45 \pm 3.65$ & 0.374 \\
\hline Nulliparous & $70(49.3 \%)$ & $76(50.0 \%)$ & 0.904 \\
\hline \multicolumn{4}{|l|}{ Fetal } \\
\hline $\begin{array}{l}\text { Gestational age } \\
\text { at birth (days) }\end{array}$ & $\begin{array}{l}279 \text { (range 238- } \\
297)\end{array}$ & $\begin{array}{l}280 \text { (range 234- } \\
297)\end{array}$ & 0.633 \\
\hline Birth weight (g) & $3593 \pm 431$ & $3603 \pm 533$ & 0.860 \\
\hline Placental weight (g) & $631 \pm 128$ & $645 \pm 142$ & 0.385 \\
\hline Fetal-placental ratio & $5.84 \pm 1.03$ & $5.74 \pm 0.98$ & 0.413 \\
\hline 5-min Apgar score & 10 (range $2-10$ ) & 9 (range 0-10) & 0.348 \\
\hline Umbilical artery pH & $7.25 \pm 0.10$ & $7.25 \pm 0.08$ & 0.831 \\
\hline $\begin{array}{l}\text { Umbilical artery } \\
\text { base excess (mmol/L) }\end{array}$ & $-4.16 \pm 3.91$ & $-4.54 \pm 3.06$ & 0.472 \\
\hline $\begin{array}{l}\text { Meconium stained } \\
\text { liquor }\end{array}$ & $29(20.4 \%)$ & $25(16.6 \%)$ & 0.443 \\
\hline Admission to NICU & $8(5.7 \%)$ & $11(7.3 \%)$ & 0.577 \\
\hline $\begin{array}{l}\text { Preterm birth, } \\
<37^{+0} \text { weeks' gestation }\end{array}$ & $1(0.7 \%)$ & $6(3.9 \%)$ & 0.068 \\
\hline Preeclampsia & $3(2.1 \%)$ & $6(3.9 \%)$ & 0.361 \\
\hline SGA/IUGR & $1(0.7 \%)$ & $3(2.0 \%)$ & 0.348 \\
\hline \multicolumn{4}{|l|}{ Mode of delivery } \\
\hline Normal & $114(80.3 \%)$ & $126(82.9 \%)$ & 0.563 \\
\hline Vacuum/forceps & $6(4.2 \%)$ & $7(4.6 \%)$ & 0.874 \\
\hline Cesarean section & $22(15.5 \%)$ & 19 (12.5\%) & 0.459 \\
\hline
\end{tabular}

Data are presented as $n$ (\%), median (range), or mean \pm SD, as appropriate

$P$ values were calculated using independent samples $t$ test for continuous variables and chi-square tests for categorical variables

NICU neonatal intensive care unit, SGA small for gestational age, IUGR intrauterine growth retardation

${ }^{\mathrm{a}} 279$ days $=39^{+6}$ weeks, 280 days $=40^{+0}$ weeks

also an association between UA Doppler indices and fetal HR $(P<0.0001)$. We found no sex differences in EFW at any gestational week, and there was no confounding effect of EFW on UA Doppler indices.

Sex-specific reference curves for the UA Doppler indices and the fetal HR for gestational weeks $20-40$ are presented in Figs. 1 and 2. The reference values with their respective 2.5 th, 5th, 10th, 25th, 50th, 75th, 90th, 95th, and 97.5th percentiles are presented in Tables 2, 3, 4, 5, 6, 7, 8, and 9 . The corresponding gestational age-related sex differences in the mean values for UA PI, RI, and S/D ratio, all adjusted for fetal HR, are displayed in Fig. 3, along with the gestational age-specific mean fetal HR for male and female fetuses during the second half of pregnancy.

The results for the gestational age-specific sex differences for fetal HR and, for the adjusted UA Doppler 

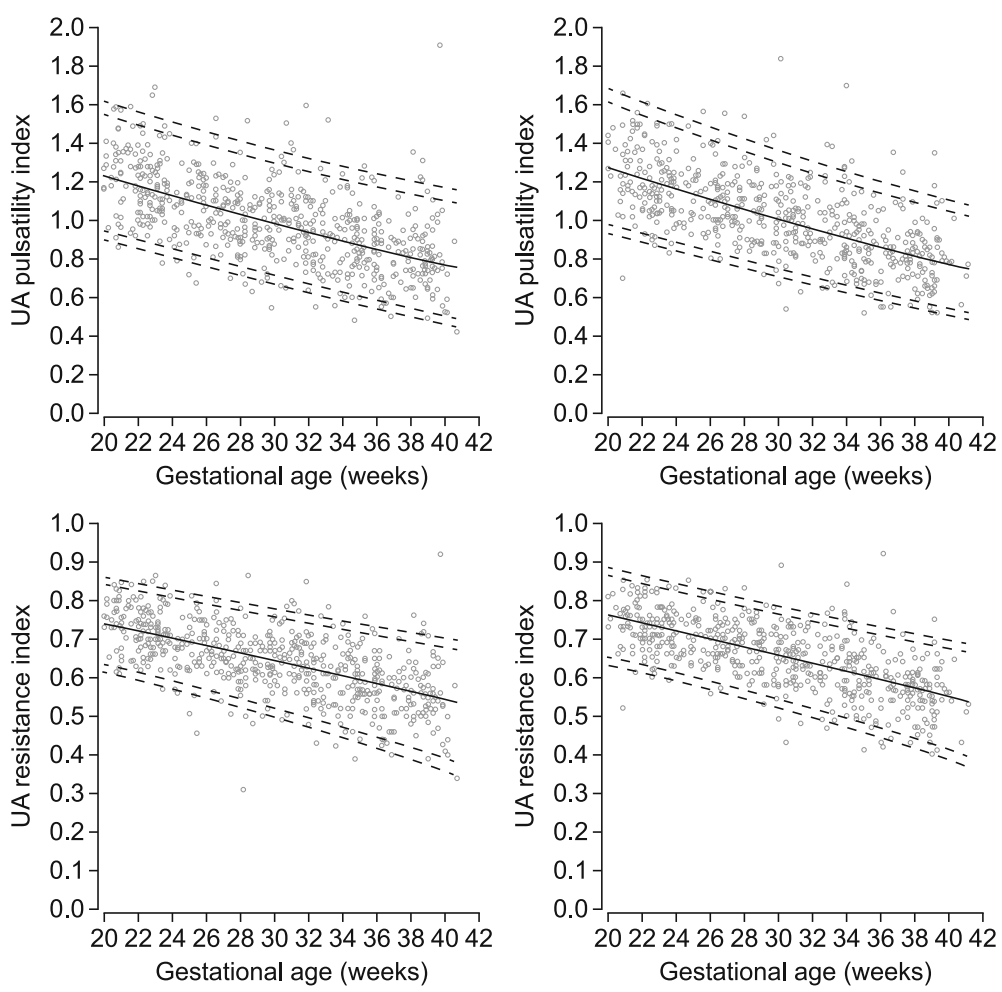

Fig. 1 Umbilical artery pulsatility index and resistance index. Sex-specific reference ranges for umbilical artery (UA) pulsatility index and resistance index (left male, right female). The solid line represents the mean, and the interrupted lines represent 2.5th, 5th, 95th, and 97.5th percentiles
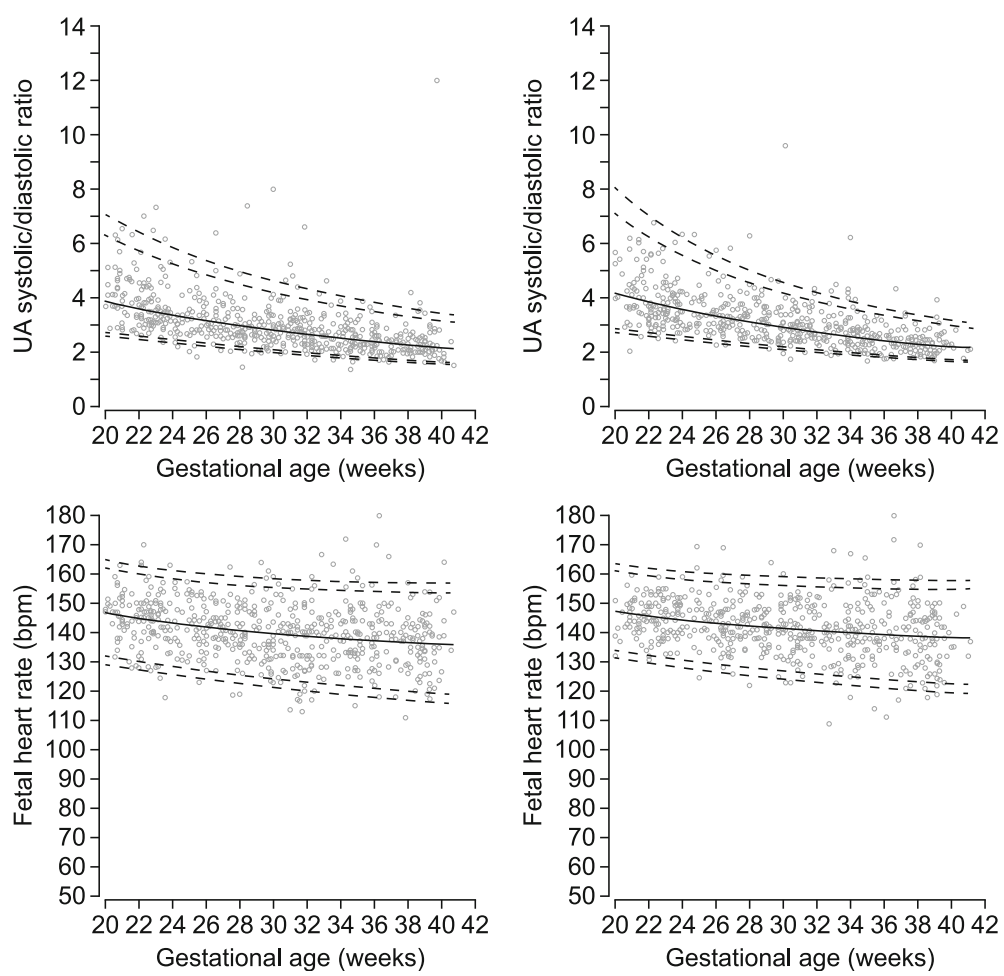

Fig. 2 Umbilical artery systolic/diastolic ratio and heart rate. Sex-specific reference ranges for umbilical artery (UA) systolic/diastolic ratio and fetal heart rate (left male, right female). The solid line represents the mean, and the interrupted lines represent 2.5th, 5th, 95th, and 97.5th percentiles 
Table 2 Umbilical artery pulsatility index (male)

\begin{tabular}{|c|c|c|c|c|c|c|c|c|c|}
\hline Gestation (week) & 2.5th percentile & 5th percentile & 10th percentile & 25th percentile & Mean & 75th percentile & 90th percentile & 95th percentile & 97.5th percentile \\
\hline 20 & 0.90 & 0.95 & 1.01 & 1.11 & 1.23 & 1.35 & 1.48 & 1.55 & 1.62 \\
\hline 21 & 0.88 & 0.92 & 0.98 & 1.08 & 1.20 & 1.33 & 1.45 & 1.52 & 1.59 \\
\hline 22 & 0.85 & 0.90 & 0.96 & 1.06 & 1.18 & 1.30 & 1.42 & 1.49 & 1.56 \\
\hline 23 & 0.83 & 0.88 & 0.93 & 1.03 & 1.15 & 1.27 & 1.39 & 1.47 & 1.53 \\
\hline 24 & 0.81 & 0.86 & 0.91 & 1.01 & 1.13 & 1.25 & 1.37 & 1.44 & 1.51 \\
\hline 25 & 0.79 & 0.83 & 0.89 & 0.98 & 1.10 & 1.22 & 1.34 & 1.42 & 1.48 \\
\hline 26 & 0.76 & 0.81 & 0.86 & 0.96 & 1.08 & 1.20 & 1.32 & 1.39 & 1.46 \\
\hline 27 & 0.74 & 0.79 & 0.84 & 0.94 & 1.05 & 1.17 & 1.29 & 1.37 & 1.43 \\
\hline 28 & 0.72 & 0.76 & 0.82 & 0.91 & 1.03 & 1.15 & 1.27 & 1.34 & 1.41 \\
\hline 29 & 0.69 & 0.74 & 0.79 & 0.89 & 1.00 & 1.13 & 1.25 & 1.32 & 1.39 \\
\hline 30 & 0.67 & 0.72 & 0.77 & 0.87 & 0.98 & 1.10 & 1.22 & 1.30 & 1.36 \\
\hline 31 & 0.65 & 0.70 & 0.75 & 0.84 & 0.96 & 1.08 & 1.20 & 1.28 & 1.34 \\
\hline 32 & 0.63 & 0.67 & 0.73 & 0.82 & 0.94 & 1.06 & 1.18 & 1.25 & 1.32 \\
\hline 33 & 0.61 & 0.65 & 0.70 & 0.80 & 0.91 & 1.04 & 1.16 & 1.23 & 1.30 \\
\hline 34 & 0.58 & 0.63 & 0.68 & 0.78 & 0.89 & 1.02 & 1.14 & 1.21 & 1.28 \\
\hline 35 & 0.56 & 0.61 & 0.66 & 0.76 & 0.87 & 1.00 & 1.12 & 1.19 & 1.26 \\
\hline 36 & 0.54 & 0.59 & 0.64 & 0.73 & 0.85 & 0.98 & 1.10 & 1.17 & 1.24 \\
\hline 37 & 0.52 & 0.57 & 0.62 & 0.71 & 0.83 & 0.96 & 1.08 & 1.16 & 1.23 \\
\hline 38 & 0.50 & 0.54 & 0.60 & 0.69 & 0.81 & 0.94 & 1.06 & 1.14 & 1.21 \\
\hline 39 & 0.48 & 0.52 & 0.58 & 0.67 & 0.79 & 0.92 & 1.04 & 1.12 & 1.19 \\
\hline 40 & 0.46 & 0.50 & 0.56 & 0.65 & 0.77 & 0.90 & 1.02 & 1.10 & 1.17 \\
\hline
\end{tabular}

Sex-specific reference values of the umbilical artery pulsatility index (UA PI) for the 2.5th, 5th, 10th, 25th, 50th, 75th, 90th, 95th, and 97.5th percentiles during the second half of pregnancy (male fetuses)

Table 3 Umbilical artery pulsatility index (female)

\begin{tabular}{|c|c|c|c|c|c|c|c|c|c|}
\hline Gestation (week) & 2.5th percentile & 5th percentile & 10th percentile & 25th percentile & Mean & 75th percentile & 90th percentile & 95th percentile & 97.5th percentile \\
\hline 20 & 0.93 & 0.98 & 1.04 & 1.15 & 1.27 & 1.41 & 1.53 & 1.61 & 1.68 \\
\hline 21 & 0.91 & 0.96 & 1.02 & 1.12 & 1.25 & 1.38 & 1.50 & 1.58 & 1.65 \\
\hline 22 & 0.89 & 0.94 & 0.99 & 1.10 & 1.22 & 1.35 & 1.47 & 1.54 & 1.61 \\
\hline 23 & 0.86 & 0.91 & 0.97 & 1.07 & 1.19 & 1.32 & 1.44 & 1.51 & 1.58 \\
\hline 24 & 0.84 & 0.89 & 0.94 & 1.04 & 1.16 & 1.29 & 1.41 & 1.48 & 1.55 \\
\hline 25 & 0.82 & 0.86 & 0.92 & 1.02 & 1.13 & 1.26 & 1.38 & 1.45 & 1.51 \\
\hline 26 & 0.80 & 0.84 & 0.90 & 0.99 & 1.11 & 1.23 & 1.35 & 1.42 & 1.48 \\
\hline 27 & 0.77 & 0.82 & 0.87 & 0.97 & 1.08 & 1.20 & 1.32 & 1.39 & 1.45 \\
\hline 28 & 0.75 & 0.80 & 0.85 & 0.94 & 1.06 & 1.17 & 1.29 & 1.36 & 1.42 \\
\hline 29 & 0.73 & 0.77 & 0.83 & 0.92 & 1.03 & 1.15 & 1.26 & 1.33 & 1.39 \\
\hline 30 & 0.71 & 0.75 & 0.80 & 0.90 & 1.00 & 1.12 & 1.23 & 1.30 & 1.37 \\
\hline 31 & 0.69 & 0.73 & 0.78 & 0.87 & 0.98 & 1.10 & 1.21 & 1.28 & 1.34 \\
\hline 32 & 0.67 & 0.71 & 0.76 & 0.85 & 0.96 & 1.07 & 1.18 & 1.25 & 1.31 \\
\hline 33 & 0.64 & 0.69 & 0.74 & 0.83 & 0.93 & 1.04 & 1.15 & 1.22 & 1.28 \\
\hline 34 & 0.62 & 0.67 & 0.71 & 0.80 & 0.91 & 1.02 & 1.13 & 1.20 & 1.26 \\
\hline 35 & 0.60 & 0.64 & 0.69 & 0.78 & 0.88 & 1.00 & 1.10 & 1.17 & 1.23 \\
\hline 36 & 0.58 & 0.62 & 0.67 & 0.76 & 0.86 & 0.97 & 1.08 & 1.15 & 1.21 \\
\hline 37 & 0.56 & 0.60 & 0.65 & 0.74 & 0.84 & 0.95 & 1.05 & 1.12 & 1.18 \\
\hline 38 & 0.54 & 0.58 & 0.63 & 0.71 & 0.82 & 0.92 & 1.03 & 1.10 & 1.16 \\
\hline 39 & 0.52 & 0.56 & 0.61 & 0.69 & 0.79 & 0.90 & 1.01 & 1.07 & 1.13 \\
\hline 40 & 0.51 & 0.54 & 0.59 & 0.67 & 0.77 & 0.88 & 0.98 & 1.05 & 1.11 \\
\hline
\end{tabular}


Table 4 Umbilical artery resistance index (male)

\begin{tabular}{|c|c|c|c|c|c|c|c|c|c|}
\hline Gestation (week) & 2.5th percentile & 5th percentile & 10th percentile & 25th percentile & Mean & 75th percentile & 90th percentile & 95th percentile & 97.5th percentile \\
\hline 20 & 0.61 & 0.64 & 0.66 & 0.70 & 0.74 & 0.78 & 0.82 & 0.84 & 0.86 \\
\hline 21 & 0.60 & 0.62 & 0.65 & 0.69 & 0.73 & 0.77 & 0.81 & 0.83 & 0.85 \\
\hline 22 & 0.59 & 0.61 & 0.64 & 0.68 & 0.72 & 0.76 & 0.80 & 0.82 & 0.84 \\
\hline 23 & 0.58 & 0.60 & 0.63 & 0.67 & 0.71 & 0.76 & 0.79 & 0.82 & 0.84 \\
\hline 24 & 0.57 & 0.59 & 0.62 & 0.66 & 0.70 & 0.75 & 0.78 & 0.81 & 0.83 \\
\hline 25 & 0.56 & 0.58 & 0.61 & 0.65 & 0.69 & 0.74 & 0.78 & 0.80 & 0.82 \\
\hline 26 & 0.55 & 0.57 & 0.60 & 0.64 & 0.68 & 0.73 & 0.77 & 0.79 & 0.81 \\
\hline 27 & 0.53 & 0.56 & 0.58 & 0.63 & 0.67 & 0.72 & 0.76 & 0.78 & 0.80 \\
\hline 28 & 0.52 & 0.55 & 0.57 & 0.62 & 0.66 & 0.71 & 0.75 & 0.77 & 0.79 \\
\hline 29 & 0.51 & 0.53 & 0.56 & 0.61 & 0.65 & 0.70 & 0.74 & 0.77 & 0.79 \\
\hline 30 & 0.50 & 0.52 & 0.55 & 0.60 & 0.64 & 0.69 & 0.73 & 0.76 & 0.78 \\
\hline 31 & 0.48 & 0.51 & 0.54 & 0.58 & 0.63 & 0.68 & 0.73 & 0.75 & 0.77 \\
\hline 32 & 0.47 & 0.50 & 0.53 & 0.57 & 0.62 & 0.67 & 0.72 & 0.74 & 0.76 \\
\hline 33 & 0.46 & 0.48 & 0.51 & 0.56 & 0.61 & 0.66 & 0.71 & 0.73 & 0.76 \\
\hline 34 & 0.44 & 0.47 & 0.50 & 0.55 & 0.60 & 0.66 & 0.70 & 0.73 & 0.75 \\
\hline 35 & 0.43 & 0.46 & 0.49 & 0.54 & 0.59 & 0.65 & 0.69 & 0.72 & 0.74 \\
\hline 36 & 0.42 & 0.45 & 0.48 & 0.53 & 0.58 & 0.64 & 0.68 & 0.71 & 0.73 \\
\hline 37 & 0.40 & 0.43 & 0.46 & 0.52 & 0.57 & 0.63 & 0.67 & 0.70 & 0.73 \\
\hline 38 & 0.39 & 0.42 & 0.45 & 0.51 & 0.56 & 0.62 & 0.67 & 0.69 & 0.72 \\
\hline 39 & 0.37 & 0.40 & 0.44 & 0.49 & 0.55 & 0.61 & 0.66 & 0.69 & 0.71 \\
\hline 40 & 0.36 & 0.39 & 0.43 & 0.48 & 0.54 & 0.60 & 0.65 & 0.68 & 0.70 \\
\hline
\end{tabular}

Sex-specific reference values of the umbilical artery resistance index (UA RI) for the 2.5th, 5th, 10th, 25th, 50th, 75th, 90th, 95th, and 97.5th percentiles during the second half of pregnancy (male fetuses)

Table 5 Umbilical artery resistance index (female)

\begin{tabular}{|c|c|c|c|c|c|c|c|c|c|}
\hline Gestation (week) & 2.5th percentile & 5th percentile & 10th percentile & 25th percentile & Mean & 75th percentile & 90th percentile & 95th percentile & 97.5th percentile \\
\hline 20 & 0.63 & 0.65 & 0.68 & 0.72 & 0.76 & 0.80 & 0.84 & 0.86 & 0.88 \\
\hline 21 & 0.62 & 0.64 & 0.67 & 0.71 & 0.75 & 0.79 & 0.83 & 0.85 & 0.87 \\
\hline 22 & 0.61 & 0.63 & 0.66 & 0.70 & 0.74 & 0.78 & 0.82 & 0.84 & 0.86 \\
\hline 23 & 0.60 & 0.62 & 0.65 & 0.69 & 0.73 & 0.77 & 0.81 & 0.83 & 0.85 \\
\hline 24 & 0.59 & 0.61 & 0.64 & 0.68 & 0.72 & 0.76 & 0.80 & 0.82 & 0.84 \\
\hline 25 & 0.58 & 0.60 & 0.62 & 0.67 & 0.71 & 0.75 & 0.79 & 0.81 & 0.83 \\
\hline 26 & 0.57 & 0.59 & 0.61 & 0.65 & 0.70 & 0.74 & 0.78 & 0.80 & 0.82 \\
\hline 27 & 0.56 & 0.58 & 0.60 & 0.64 & 0.69 & 0.73 & 0.77 & 0.79 & 0.81 \\
\hline 28 & 0.54 & 0.57 & 0.59 & 0.63 & 0.68 & 0.72 & 0.76 & 0.78 & 0.80 \\
\hline 29 & 0.53 & 0.56 & 0.58 & 0.62 & 0.67 & 0.71 & 0.75 & 0.77 & 0.79 \\
\hline 30 & 0.52 & 0.54 & 0.57 & 0.61 & 0.66 & 0.70 & 0.74 & 0.76 & 0.78 \\
\hline 31 & 0.51 & 0.53 & 0.56 & 0.60 & 0.65 & 0.69 & 0.73 & 0.75 & 0.77 \\
\hline 32 & 0.50 & 0.52 & 0.55 & 0.59 & 0.64 & 0.68 & 0.72 & 0.74 & 0.76 \\
\hline 33 & 0.48 & 0.51 & 0.53 & 0.58 & 0.63 & 0.67 & 0.71 & 0.74 & 0.76 \\
\hline 34 & 0.47 & 0.49 & 0.52 & 0.57 & 0.61 & 0.66 & 0.70 & 0.73 & 0.75 \\
\hline 35 & 0.46 & 0.48 & 0.51 & 0.55 & 0.60 & 0.65 & 0.69 & 0.72 & 0.74 \\
\hline 36 & 0.44 & 0.47 & 0.50 & 0.54 & 0.59 & 0.64 & 0.68 & 0.71 & 0.73 \\
\hline 37 & 0.43 & 0.45 & 0.48 & 0.53 & 0.58 & 0.63 & 0.67 & 0.70 & 0.72 \\
\hline 38 & 0.41 & 0.44 & 0.47 & 0.52 & 0.57 & 0.62 & 0.66 & 0.69 & 0.71 \\
\hline 39 & 0.40 & 0.43 & 0.46 & 0.51 & 0.56 & 0.61 & 0.65 & 0.68 & 0.70 \\
\hline 40 & 0.38 & 0.41 & 0.44 & 0.49 & 0.55 & 0.60 & 0.65 & 0.67 & 0.69 \\
\hline
\end{tabular}

Sex-specific reference values of the umbilical artery resistance index (UA RI) for the 2.5th, 5th, 10th, 25th, 50th, 75th, 90th, 95th, and 97.5th percentiles during the second half of pregnancy (female fetuses) 
Table 6 Umbilical artery systolic/diastolic ratio (male)

\begin{tabular}{|c|c|c|c|c|c|c|c|c|c|}
\hline Gestation (week) & 2.5th percentile & 5th percentile & 10th percentile & 25th percentile & Mean & 75th percentile & 90th percentile & 95th percentile & 97.5th percentile \\
\hline 20 & 2.6 & 2.7 & 2.9 & 3.3 & 3.9 & 4.6 & 5.5 & 6.3 & 7.1 \\
\hline 21 & 2.5 & 2.7 & 2.8 & 3.2 & 3.7 & 4.4 & 5.3 & 6.0 & 6.7 \\
\hline 22 & 2.4 & 2.6 & 2.8 & 3.1 & 3.6 & 4.3 & 5.1 & 5.7 & 6.4 \\
\hline 23 & 2.4 & 2.5 & 2.7 & 3.0 & 3.5 & 4.1 & 4.9 & 5.5 & 6.1 \\
\hline 24 & 2.3 & 2.4 & 2.6 & 2.9 & 3.4 & 4.0 & 4.7 & 5.3 & 5.8 \\
\hline 25 & 2.2 & 2.4 & 2.5 & 2.8 & 3.3 & 3.8 & 4.5 & 5.0 & 5.6 \\
\hline 26 & 2.2 & 2.3 & 2.5 & 2.8 & 3.2 & 3.7 & 4.4 & 4.9 & 5.4 \\
\hline 27 & 2.1 & 2.2 & 2.4 & 2.7 & 3.1 & 3.6 & 4.2 & 4.7 & 5.2 \\
\hline 28 & 2.1 & 2.2 & 2.3 & 2.6 & 3.0 & 3.5 & 4.1 & 4.5 & 5.0 \\
\hline 29 & 2.0 & 2.1 & 2.3 & 2.5 & 2.9 & 3.4 & 3.9 & 4.3 & 4.8 \\
\hline 30 & 2.0 & 2.1 & 2.2 & 2.5 & 2.8 & 3.3 & 3.8 & 4.2 & 4.6 \\
\hline 31 & 1.9 & 2.0 & 2.1 & 2.4 & 2.7 & 3.2 & 3.7 & 4.1 & 4.5 \\
\hline 32 & 1.9 & 2.0 & 2.1 & 2.3 & 2.7 & 3.1 & 3.6 & 3.9 & 4.3 \\
\hline 33 & 1.8 & 1.9 & 2.0 & 2.3 & 2.6 & 3.0 & 3.5 & 3.8 & 4.2 \\
\hline 34 & 1.8 & 1.9 & 2.0 & 2.2 & 2.5 & 2.9 & 3.4 & 3.7 & 4.1 \\
\hline 35 & 1.7 & 1.8 & 1.9 & 2.2 & 2.5 & 2.8 & 3.3 & 3.6 & 3.9 \\
\hline 36 & 1.7 & 1.8 & 1.9 & 2.1 & 2.4 & 2.8 & 3.2 & 3.5 & 3.8 \\
\hline 37 & 1.7 & 1.8 & 1.9 & 2.1 & 2.3 & 2.7 & 3.1 & 3.4 & 3.7 \\
\hline 38 & 1.6 & 1.7 & 1.8 & 2.0 & 2.3 & 2.6 & 3.0 & 3.3 & 3.6 \\
\hline 39 & 1.6 & 1.7 & 1.8 & 2.0 & 2.2 & 2.6 & 2.9 & 3.2 & 3.5 \\
\hline 40 & 1.6 & 1.6 & 1.7 & 1.9 & 2.2 & 2.5 & 2.9 & 3.1 & 3.4 \\
\hline
\end{tabular}

Sex-specific reference values of the umbilical artery systolic/diastolic (UA S/D) ratio for the 2.5th, 5th, 10th, 25th, 50th, 75th, 90th, 95th, and 97.5th percentiles during the second half of pregnancy (male fetuses)

Table 7 Umbilical artery systolic/diastolic ratio (female)

\begin{tabular}{|c|c|c|c|c|c|c|c|c|c|}
\hline Gestation (week) & 2.5th percentile & 5th percentile & 10th percentile & 25th percentile & Mean & 75th percentile & 90th percentile & 95th percentile & 97.5th percentile \\
\hline 20 & 2.7 & 2.9 & 3.1 & 3.5 & 4.2 & 5.0 & 6.2 & 7.1 & 8.1 \\
\hline 21 & 2.6 & 2.8 & 3.0 & 3.4 & 4.0 & 4.8 & 5.8 & 6.6 & 7.5 \\
\hline 22 & 2.6 & 2.7 & 2.9 & 3.3 & 3.8 & 4.6 & 5.5 & 6.2 & 7.0 \\
\hline 23 & 2.5 & 2.6 & 2.8 & 3.2 & 3.7 & 4.4 & 5.2 & 5.9 & 6.6 \\
\hline 24 & 2.4 & 2.6 & 2.7 & 3.1 & 3.6 & 4.2 & 5.0 & 5.6 & 6.2 \\
\hline 25 & 2.4 & 2.5 & 2.7 & 3.0 & 3.4 & 4.0 & 4.7 & 5.3 & 5.8 \\
\hline 26 & 2.3 & 2.4 & 2.6 & 2.9 & 3.3 & 3.9 & 4.5 & 5.0 & 5.5 \\
\hline 27 & 2.3 & 2.4 & 2.5 & 2.8 & 3.2 & 3.7 & 4.3 & 4.8 & 5.3 \\
\hline 28 & 2.2 & 2.3 & 2.5 & 2.7 & 3.1 & 3.6 & 4.2 & 4.6 & 5.0 \\
\hline 29 & 2.1 & 2.3 & 2.4 & 2.6 & 3.0 & 3.5 & 4.0 & 4.4 & 4.8 \\
\hline 30 & 2.1 & 2.2 & 2.3 & 2.6 & 2.9 & 3.3 & 3.8 & 4.2 & 4.6 \\
\hline 31 & 2.0 & 2.1 & 2.3 & 2.5 & 2.8 & 3.2 & 3.7 & 4.0 & 4.4 \\
\hline 32 & 2.0 & 2.1 & 2.2 & 2.4 & 2.7 & 3.1 & 3.6 & 3.9 & 4.2 \\
\hline 33 & 1.9 & 2.0 & 2.2 & 2.4 & 2.7 & 3.0 & 3.4 & 3.7 & 4.0 \\
\hline 34 & 1.9 & 2.0 & 2.1 & 2.3 & 2.6 & 2.9 & 3.3 & 3.6 & 3.9 \\
\hline 35 & 1.9 & 1.9 & 2.0 & 2.2 & 2.5 & 2.8 & 3.2 & 3.5 & 3.7 \\
\hline 36 & 1.8 & 1.9 & 2.0 & 2.2 & 2.4 & 2.8 & 3.1 & 3.4 & 3.6 \\
\hline 37 & 1.8 & 1.9 & 2.0 & 2.1 & 2.4 & 2.7 & 3.0 & 3.3 & 3.5 \\
\hline 38 & 1.7 & 1.8 & 1.9 & 2.1 & 2.3 & 2.6 & 2.9 & 3.2 & 3.4 \\
\hline 39 & 1.7 & 1.8 & 1.9 & 2.0 & 2.3 & 2.5 & 2.8 & 3.1 & 3.3 \\
\hline 40 & 1.7 & 1.7 & 1.8 & 2.0 & 2.2 & 2.5 & 2.8 & 3.0 & 3.2 \\
\hline
\end{tabular}


Table 8 Fetal heart rate (male), beats per minute

\begin{tabular}{|c|c|c|c|c|c|c|c|c|c|}
\hline Gestation (week) & 2.5th percentile & 5th percentile & 10th percentile & 25th percentile & Mean & 75th percentile & 90th percentile & 95th percentile & 97.5th percentile \\
\hline 20 & 129 & 132 & 135 & 141 & 147 & 153 & 159 & 162 & 165 \\
\hline 21 & 128 & 131 & 134 & 140 & 146 & 152 & 157 & 161 & 164 \\
\hline 22 & 127 & 130 & 133 & 139 & 145 & 151 & 156 & 160 & 163 \\
\hline 23 & 127 & 129 & 133 & 138 & 144 & 150 & 156 & 159 & 162 \\
\hline 24 & 126 & 129 & 132 & 137 & 143 & 149 & 155 & 158 & 161 \\
\hline 25 & 125 & 128 & 131 & 136 & 142 & 149 & 154 & 158 & 160 \\
\hline 26 & 124 & 127 & 130 & 136 & 142 & 148 & 154 & 157 & 160 \\
\hline 27 & 123 & 126 & 130 & 135 & 141 & 147 & 153 & 156 & 159 \\
\hline 28 & 123 & 126 & 129 & 134 & 141 & 147 & 153 & 156 & 159 \\
\hline 29 & 122 & 125 & 128 & 134 & 140 & 146 & 152 & 156 & 159 \\
\hline 30 & 121 & 124 & 128 & 133 & 140 & 146 & 152 & 155 & 158 \\
\hline 31 & 121 & 124 & 127 & 133 & 139 & 146 & 152 & 155 & 158 \\
\hline 32 & 120 & 123 & 127 & 132 & 139 & 145 & 151 & 155 & 158 \\
\hline 33 & 120 & 123 & 126 & 132 & 138 & 145 & 151 & 155 & 158 \\
\hline 34 & 119 & 122 & 125 & 131 & 138 & 145 & 151 & 154 & 158 \\
\hline 35 & 118 & 121 & 125 & 131 & 138 & 144 & 151 & 154 & 157 \\
\hline 36 & 118 & 121 & 125 & 131 & 137 & 144 & 150 & 154 & 157 \\
\hline 37 & 117 & 120 & 124 & 130 & 137 & 144 & 150 & 154 & 157 \\
\hline 38 & 117 & 120 & 124 & 130 & 137 & 144 & 150 & 154 & 157 \\
\hline 39 & 116 & 120 & 123 & 129 & 136 & 143 & 150 & 154 & 157 \\
\hline 40 & 116 & 119 & 123 & 129 & 136 & 143 & 150 & 154 & 157 \\
\hline
\end{tabular}

Sex-specific reference values of the fetal heart rate (HR) for the 2.5th, 5th, 10th, 25th, 50th, 75th, 90th, 95th, and 97.5th percentiles during the second half of pregnancy (male fetuses)

Table 9 Fetal heart rate (female), beats per minute

\begin{tabular}{|c|c|c|c|c|c|c|c|c|c|}
\hline Gestation (week) & 2.5th percentile & 5th percentile & 10th percentile & 25th percentile & Mean & 75th percentile & 90th percentile & 95th percentile & 97.5th percentile \\
\hline 20 & 131 & 134 & 137 & 142 & 147 & 153 & 158 & 161 & 164 \\
\hline 21 & 131 & 133 & 136 & 141 & 146 & 152 & 157 & 160 & 163 \\
\hline 22 & 130 & 132 & 135 & 140 & 146 & 151 & 156 & 159 & 162 \\
\hline 23 & 129 & 132 & 134 & 139 & 145 & 151 & 156 & 159 & 161 \\
\hline 24 & 128 & 131 & 134 & 139 & 144 & 150 & 155 & 158 & 161 \\
\hline 25 & 127 & 130 & 133 & 138 & 144 & 150 & 155 & 158 & 161 \\
\hline 26 & 127 & 129 & 132 & 138 & 143 & 149 & 154 & 157 & 160 \\
\hline 27 & 126 & 129 & 132 & 137 & 143 & 149 & 154 & 157 & 160 \\
\hline 28 & 125 & 128 & 131 & 136 & 142 & 148 & 154 & 157 & 160 \\
\hline 29 & 125 & 127 & 131 & 136 & 142 & 148 & 153 & 156 & 159 \\
\hline 30 & 124 & 127 & 130 & 135 & 141 & 147 & 153 & 156 & 159 \\
\hline 31 & 124 & 126 & 130 & 135 & 141 & 147 & 153 & 156 & 159 \\
\hline 32 & 123 & 126 & 129 & 135 & 141 & 147 & 152 & 156 & 159 \\
\hline 33 & 123 & 125 & 129 & 134 & 140 & 147 & 152 & 156 & 159 \\
\hline 34 & 122 & 125 & 128 & 134 & 140 & 146 & 152 & 156 & 159 \\
\hline 35 & 122 & 124 & 128 & 133 & 140 & 146 & 152 & 155 & 158 \\
\hline 36 & 121 & 124 & 127 & 133 & 139 & 146 & 152 & 155 & 158 \\
\hline 37 & 121 & 124 & 127 & 133 & 139 & 146 & 152 & 155 & 158 \\
\hline 38 & 120 & 123 & 127 & 132 & 139 & 145 & 151 & 155 & 158 \\
\hline 39 & 120 & 123 & 126 & 132 & 139 & 145 & 151 & 155 & 158 \\
\hline 40 & 120 & 123 & 126 & 132 & 138 & 145 & 151 & 155 & 158 \\
\hline
\end{tabular}



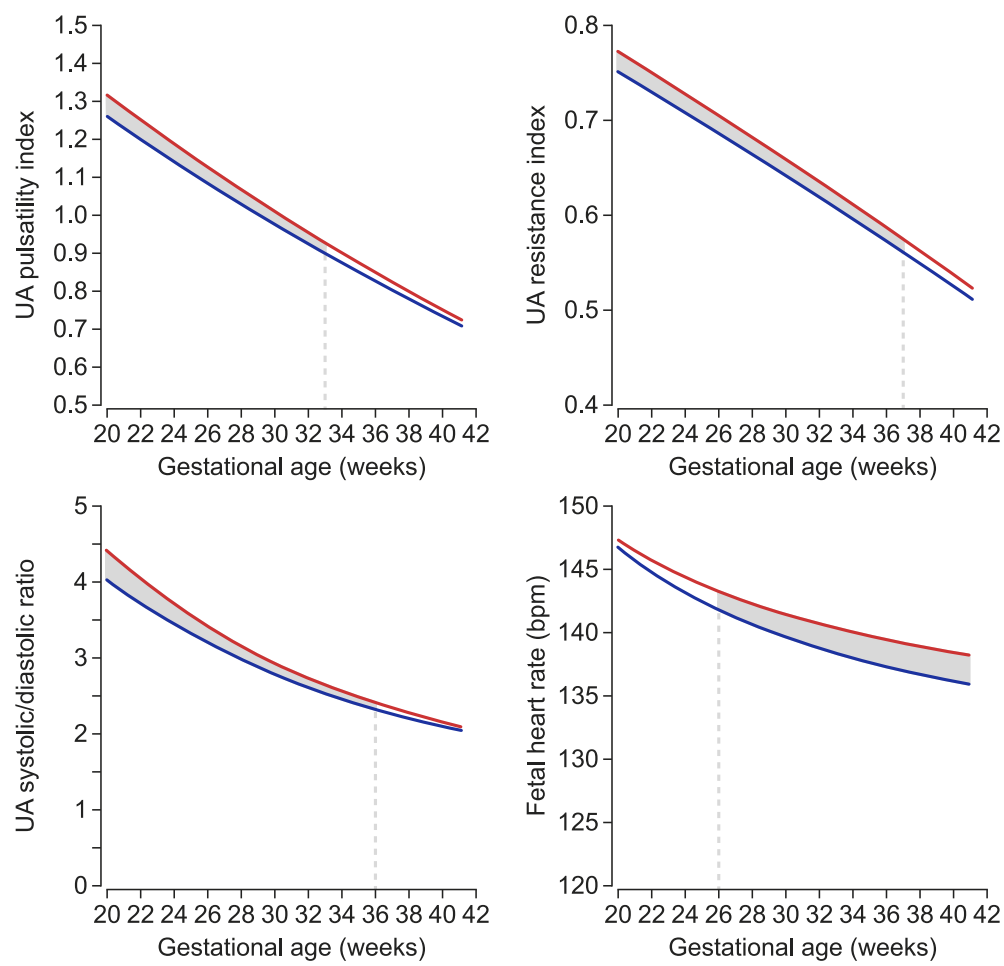

Fig. 3 Sex differences in fetal heart rate and umbilical artery Doppler indices adjusted for fetal heart rate. Gestational age-related sex differences in the mean values for umbilical artery (UA) pulsatility index (top left), resistance index (top right), systolic/diastolic ratio (bottom left), all adjusted for fetal heart rate, and fetal heart rate (bottom right) during the second half of pregnancy. The red line represents female, and the blue line represents male. The shaded area indicates significant differences $(P<0.05)$

indices, are shown in Table 10. We found significant differences in UA PI, RI, S/D ratio, and HR between male and female fetuses. Female fetuses had significantly higher values for UA PI (range 2.1-4.2\%), RI (range 1.7-3.3\%), and S/D ratio (range $4.0-8.1 \%$ ) from $20^{+0}$ weeks to $32^{+6}$ and $36^{+6}$ and $35^{+6}$ weeks, respectively, but equalized towards term ( 40 weeks of gestation). For fetal HR, the mean values were similar between male and female fetuses from $20^{+0}$ to $25^{+6}$ weeks, but a divergent trend was observed thereafter with the female fetuses showing higher HR (range 0.7-2.2\%) compared with male fetuses.

\section{Discussion}

The present longitudinal study has demonstrated significant sex differences in UA Doppler indices, female fetuses having significantly more pulsatile waveform than male fetuses during gestational weeks $20^{+0}-36^{+6}$ but not thereafter. The magnitude of effect ranged between 2.1 and $4.2 \%$ for the UA PI. Correspondingly, the study provided sex-specific reference ranges for 20-40 weeks' gestation for the most commonly used indices. As for the fetal $H R$, the pattern was different; male and female fetuses had similar HR from $20^{+0}$ to $25^{+6}$ weeks, but thereafter, the female fetuses had significantly higher HR.
The strength of the study is its longitudinal design and a relatively large sample size (650 observations for male and 611 for female fetuses) providing sufficient power to discover significant sex differences and to construct robust sex-specific reference ranges. The prospective longitudinal design with serial measurements at reasonably spaced intervals during pregnancy is preferable to a cross-sectional design for constructing reference intervals since it better reflects the development during gestation and, in our case, improves the precision of individual participants' observations. The limitations of our study are related to technical issues concerning UA Doppler velocimetry, and the data being collected from three separate studies, with different operators. However, all the measurements were obtained at a free loop of umbilical cord, under fetal quiescence, keeping the angle of insonation as low as possible (always $<15^{\circ}$ ). The intra-observer CV for UA PI, RI, and S/D ratio were 10 . $5,6.8$, and $13.0 \%$, respectively [26].

This study confirms the findings of previous crosssectional studies that report sex differences in UA Doppler indices during the second and third trimester of pregnancy $[31,32]$ and that these differences tapered off towards term [30]. However, we were not able to establish at what time in gestation these differences emerged. 
Table 10 Level of significance for sex differences in umbilical artery Doppler indices and fetal heart rate

\begin{tabular}{|c|c|c|c|c|}
\hline \multirow{2}{*}{$\begin{array}{l}\text { Gestation } \\
\text { (week) }\end{array}$} & \multirow{2}{*}{$\begin{array}{l}\text { UA PI } \\
P \text { value }\end{array}$} & \multirow{2}{*}{$\begin{array}{l}\text { UA RI }{ }^{\mathrm{a}} \\
P \text { value }\end{array}$} & \multirow{2}{*}{$\begin{array}{l}\text { UA S/D ratio } \\
P \text { value }\end{array}$} & \multirow{2}{*}{$\begin{array}{l}\text { Fetal } \mathrm{HR} \\
P \text { value }\end{array}$} \\
\hline & & & & \\
\hline 20 & 0.00795 & 0.00282 & 0.00213 & 0.58551 \\
\hline 21 & 0.00695 & 0.00219 & 0.00171 & 0.44020 \\
\hline 22 & 0.00621 & 0.00172 & 0.00139 & 0.30634 \\
\hline 23 & 0.00573 & 0.00139 & 0.00117 & 0.19662 \\
\hline 24 & 0.00552 & 0.00117 & 0.00103 & 0.11804 \\
\hline 25 & 0.00564 & 0.00104 & 0.00097 & 0.06888 \\
\hline 26 & 0.00616 & 0.00102 & 0.00100 & 0.04136 \\
\hline 27 & 0.00724 & 0.00109 & 0.00113 & 0.02704 \\
\hline 28 & 0.00915 & 0.00132 & 0.00141 & 0.01991 \\
\hline 29 & 0.01234 & 0.00178 & 0.00196 & 0.01656 \\
\hline 30 & 0.01748 & 0.00264 & 0.00295 & 0.01526 \\
\hline 31 & 0.02550 & 0.00421 & 0.00473 & 0.01518 \\
\hline 32 & 0.03755 & 0.00699 & 0.00784 & 0.01586 \\
\hline 33 & 0.05486 & 0.01172 & 0.01309 & 0.01706 \\
\hline 34 & 0.07849 & 0.01939 & 0.02150 & 0.01864 \\
\hline 35 & 0.10911 & 0.03110 & 0.03424 & 0.02049 \\
\hline 36 & 0.14679 & 0.04786 & 0.05239 & 0.02253 \\
\hline 37 & 0.19098 & 0.07041 & 0.07670 & 0.02470 \\
\hline 38 & 0.24064 & 0.09901 & 0.10746 & 0.02696 \\
\hline 39 & 0.29443 & 0.13342 & 0.14444 & 0.02926 \\
\hline 40 & 0.35092 & 0.17300 & 0.18692 & 0.03159 \\
\hline Overall ${ }^{b}$ & 0.07560 & 0.01850 & 0.01980 & 0.02560 \\
\hline
\end{tabular}

The results for the gestational age-specific sex differences in mean values for fetal heart rate (HR) and for the adjusted umbilical artery (UA) Doppler indices, organized by gestational week

$U A$ umbilical artery, $P I$ pulsatility index, $R /$ resistance index, $S / D$ ratio systolic/ diastolic ratio, $H R$ heart rate

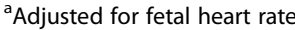

${ }^{b}$ Overall level of significance for sex differences during 20-40 weeks of gestation

It would have been desirable to have serial measurements starting from early pregnancy.

Our findings add weight to the recognition of sex differences in fetal development and adaption to the intrauterine environment. The male disadvantage in perinatal outcome when it comes to fetal distress during labor [3, 4], premature birth [5, 6], adverse neonatal outcome [7], and early neonatal death [2] is well documented. It is also well documented that there are significant sex differences in growth of estimated fetal weight [19], birth weight, and placental weight [13], and male and female fetuses have significant differences in growth patterns of individual biometric measurements [41]. Such differences in growth dynamics corroborate the findings of Orzack et al. [10] who found that the unbiased male/female ratio at conception had increased at birth due to a higher female mortality during pregnancy. However, male and female mortality during pregnancy had temporal differences causing undulations in the sex ratio. These findings constitute a plethora of details in which our circulatory results add another piece of evidence to sex differentiation being reflected in all organ systems.

With this background, our finding that there was no significant effect of fetal sex on fetal growth (i.e., EFW) is unexpected, as a recent multinational study showed that fetal sex had an effect of 3.5-4.5\% on EFW [42]. However, that study had a considerably higher power than our present study. One can therefore speculate that the present finding of no sex effect on fetal weight could be due to chance, or, as shown in the recent WHO study, due to variations in growth patterns. However, the negligible $(10 \mathrm{~g})$ difference in birth weight we observed between the sexes (3593 vs. 3603 g) corroborates our intrauterine growth estimates and ensures that the effect on the Doppler indices was due to sex differences. The issue is important because a difference in size could possibly have explained some of the results. It is interesting, however, that a previous study found "no meaningful correlation between fetal weight and impedance indices" [43].

Mechanisms associated with potential male susceptibility are difficult to underpin. Male fetuses appear to prioritize growth to a greater extent than females and continue to grow in spite of unfavorable intrauterine environment [14]. This may put them at higher risk due to lack of reserve. A higher UA PI, as we have observed in females, could result in a reduction in fetal growth velocity and thereby reduce the risk of adverse outcomes. The mechanisms behind the observed differences in UA Doppler indices are not clear. Slightly higher UA Doppler indices cannot be equated to reduced placental function, and these differences were less pronounced close to term. However, it has been shown that male fetuses born at 24-28 weeks of gestation have more peripheral vasodilatation compared to female fetuses [44]. Furthermore, pregnant women carrying male fetuses are reported to have higher angiotensin (Ang) 1-7 to Ang II ratio in the second trimester [45]. As Ang II is a potent vasoconstrictor and Ang 1-7 is a known vasodilator, relative vasodilatation of placental vessels could be responsible for lower UA PI, RI, and S/D ratio observed in male fetuses.

UA Doppler indices, a surrogate for placental impedance [46], have proved valuable in assessing fetal wellbeing and have the potential to save lives [23]. However, these relations are not consistent [47], as shown in sheep experiments $[48,49]$. Although PI increases when embolization causes reduction in vascular cross-section, comparable reduction in vascular cross-section due to angiotensin II did not increase the PI and could even decrease the PI while vascular resistance increased. The reason for this may be a difference in vessel geometry that could impact the wave reflection, a major modifier of the arterial waveform [50, 
51]. Thus, the exact mechanism behind the sex difference in the UA pulsatility is not certain.

Another significant finding in the present study was the relatively higher HR in female compared with male fetuses after $26^{+0}$ weeks of gestation, a difference that increased with gestational age (Fig. 3). Higher HR among female fetuses has also been reported previously by others [31, 52]. A plausible cause for having different heart rates in male and female fetuses is differences in hormone levels and rate of maturation of their autonomic nervous system. Higher heart rate variability [53], more complex heart rate patterns [54], and higher catecholamine levels observed in female compared to male fetuses could explain these differences.

In fetal sheep experiments, Morrow et al. demonstrated a significant inverse correlation between the UA Doppler indices (PI, RI, and S/D ratio) and HR [51]. When the HR increased, the UA Doppler indices decreased. We found both higher HR and UA PI, RI, and S/D ratio in female fetuses compared to males, but while the sex differences in HR increased as the pregnancy advanced, the sex differences in the Doppler indices decreased and ceased to exist by term. When we adjusted the gestational age-related sex differences in the mean values for UA PI, RI, and S/D ratio for the fetal HR, the effect size actually increased, i.e., the sexual dimorphism in the UA Doppler indices became more prominent.

Several studies have shown a male preponderance when abnormal UA Doppler waveform is used as a marker of placental dysfunction in pregnancies with IUGR $[55,56]$. Increased UA PI correlates with reduced feto-placental perfusion [57] and the degree of microvascular lesions in the placenta [24]. Use of sex-specific reference intervals of UA Doppler indices could potentially improve the identification of pregnancies with placental dysfunction.

\section{Conclusions}

We have demonstrated gestational age-dependent sex differences in UA Doppler indices during the second half of physiological pregnancies and therefore established sex-specific reference ranges. Although the sex difference is modest $(2-8 \%)$, we believe such references are useful for refining prediction and monitoring of risk pregnancies at a time when such parameters easily are added into software applications increasingly used in clinical practice, particularly since individualized diagnostics and management is an issue.

\section{Abbreviations}

AC: Abdominal circumference; ALARA: As low as reasonably achievable; BMI: Body mass index; CV: Coefficient of variation; EDV: End-diastolic velocity; EFW: Estimated fetal weight; FL: Femur length; HR: Heart rate; IUGR: Intrauterine growth retardation; MCA: Middle cerebral artery; PI: Pulsatility index; PSV: Peak systolic velocity; Quv: Umbilical venous blood flow; Rl: Resistance index; S/D ratio: Systolic/diastolic ratio; SD: Standard deviation; SGA: Small for gestational age; TAMXV: Time-averaged maximum velocity; UA: Umbilical artery

\section{Acknowledgements}

We would like to thank Åse Vårtun, Bodil Hvingel, and Norbert Szunyogh for their help with patient recruitment and examination.

\section{Funding}

This research was supported by the Regional Health Authority of North Norway. The publication charges for this article have been funded by a grant from the publication fund of UiT The Arctic University of Norway. The funders had no role in designing the study, collection, analysis and interpretation of data, writing of the report, or in the decision to submit the article for publication.

\section{Availability of data and materials}

The datasets generated and analyzed during the current study are available from the corresponding author on reasonable request.

\section{Authors' contributions}

$\mathrm{CW}$, TK, and GA participated in the conception and design of the experiments. $\mathrm{KF}$ and $\mathrm{GA}$ recruited the patients and performed the ultrasound examinations. $\mathrm{CW}, \mathrm{KF}$, and GA participated in the collection of data. CW and TW performed the statistical analysis. CW, TK, and GA made substantial contributions to the interpretation of the data and writing of the manuscript. All authors read and approved the final manuscript.

\section{Ethics approval and consent to participate}

The study protocols were approved by the Regional Committee for Medical and Health Research Ethics-North Norway (REK Nord 74/2001, 52/2005, and 105/2008) and an informed written consent was obtained from each participant.

\section{Consent for publication}

Not applicable.

\section{Competing interests}

The authors declare that they have no competing interests.

\section{Publisher's Note}

Springer Nature remains neutral with regard to jurisdictional claims in published maps and institutional affiliations.

\section{Author details}

${ }^{1}$ Women's Health and Perinatology Research Group, Department of Clinical Medicine, Faculty of Health Sciences, UiT-The Arctic University of Norway, Tromso, Norway. ${ }^{2}$ Department of Obstetrics and Gynecology, University Hospital of North Norway, Sykehusveien 38, PO Box 24, N-9038 Tromso, Norway. ${ }^{3}$ Department of Community Medicine, Faculty of Health Sciences, UiT-The Arctic University of Norway, Tromso, Norway. ${ }^{4}$ Department of Clinical Science, University of Bergen, Bergen, Norway. ${ }^{5}$ Department of Obstetrics and Gynecology, Haukeland University Hospital, Bergen, Norway.

${ }^{6}$ Department of Clinical Science, Intervention and Technology, Karolinska Institute, Stockholm, Sweden.

Received: 29 December 2017 Accepted: 2 April 2018

Published online: 18 April 2018

\section{References}

1. Institute of Medicine, Committee on Understanding the Biology of Sex and Gender Differences. Exploring the biological contributions to human health: does sex matter? Washington, DC: National Academies Press (US); 2001.

2. Di Renzo GC, Rosati A, Sarti RD, Cruciani L, Cutuli AM. Does fetal sex affect pregnancy outcome? Gend Med. 2007:4:19-30.

3. Dunn L, Prior T, Greer R, Kumar S. Gender specific intrapartum and neonatal outcomes for term babies. Eur J Obstet Gynecol Reprod Biol. 2015;185:19-22.

4. Bekedam DJ, Engelsbel S, Mol BW, Buitendijk SE, van der Pal-de Bruin KM. Male predominance in fetal distress during labor. Am J Obstet Gynecol. 2002;187:1605-7.

5. Peelen MJCS, Kazemier BM, Ravelli ACJ, De Groot CJM, Van Der Post JAM, Mol BWJ, Hajenius PJ, Kok M. Impact of fetal gender on the risk of preterm birth, a national cohort study. Acta Obstet Gynecol Scand. 2016;95:1034-41. 
6. Zeitlin J, Saurel-Cubizolles MJ, De Mouzon J, Rivera L, Ancel PY, Blondel B, Kaminski M. Fetal sex and preterm birth: are males at greater risk? Hum Reprod. 2002;17:2762-8.

7. Peacock JL, Marston L, Marlow N, Calvert SA, Greenough A. Neonatal and infant outcome in boys and girls born very prematurely. Pediatr Res. 2012;71:305-10.

8. Naeye RL, Burt LS, Wright DL, Blanc WA, Tatter D. Neonatal mortality, the male disadvantage. Pediatrics. 1971;48:902-6.

9. Naeye RL, Demers LM. Differing effects of fetal sex on pregnancy and its outcome. Am J Med Genet Suppl. 1987;3:67-74.

10. Orzack SH, Stubblefield JW, Akmaev VR, Colls P, Munne S, Scholl T, Steinsaltz $D$, Zuckerman JE. The human sex ratio from conception to birth. Proc Natl Acad Sci U S A. 2015;112:E2102-11.

11. Brown ZA, Schalekamp-Timmermans S, Tiemeier HW, Hofman A, Jaddoe W, Steegers EA. Fetal sex specific differences in human placentation: a prospective cohort study. Placenta. 2014;35:359-64.

12. Rosenfeld CS. Sex-specific placental responses in fetal development Endocrinology. 2015;156:3422-34

13. Wallace JM, Bhattacharya S, Horgan GW. Gestational age, gender and parity specific centile charts for placental weight for singleton deliveries in Aberdeen, UK. Placenta. 2013;34:269-74.

14. Clifton VL. Review: sex and the human placenta: mediating differential strategies of fetal growth and survival. Placenta. 2010;31(Suppl):S33-9.

15. Cvitic S, Longtine MS, Hackl H, Wagner K, Nelson MD, Desoye G, Hiden U. The human placental sexome differs between trophoblast epithelium and villous vessel endothelium. PLoS One. 2013;8:e79233.

16. Ghidini A, Salafia CM. Gender differences of placental dysfunction in severe prematurity. BJOG. 2005;112:140-4.

17. Challis J, Newnham J, Petraglia F, Yeganegi M, Bocking A. Fetal sex and preterm birth. Placenta. 2013;34:95-9.

18. Gabory A, Roseboom TJ, Moore T, Moore LG, Junien C. Placental contribution to the origins of sexual dimorphism in health and diseases: sex chromosomes and epigenetics. Biol Sex Differ. 2013:4:5.

19. Johnsen SL, Rasmussen S, Wilsgaard T, Sollien R, Kiserud T. Longitudinal reference ranges for estimated fetal weight. Acta Obstet Gynecol Scand. 2006;85:286-97.

20. Rizzo G, Prefumo F, Ferrazzi E, Zanardini C, Di Martino D, Boito S, Aiello E, Ghi T. The effect of fetal sex on customized fetal growth charts. J Matern Fetal Neonatal Med. 2016;29:3768-75.

21. Trudell AS, Cahill AG, Tuuli MG, Macones GA, Odibo AO. Stillbirth and the small fetus: use of a sex-specific versus a non-sex-specific growth standard. J Perinatol. 2015;35:566-9.

22. Marsal K. Obstetric management of intrauterine growth restriction. Best Pract Res Clin Obstet Gynaecol. 2009;23:857-70.

23. Alfirevic Z, Stampalija T, Gyte GM. Fetal and umbilical Doppler ultrasound in high-risk pregnancies. Cochrane Database Syst Rev. 2013;11:Cd007529.

24. Giles WB, Trudinger BJ, Baird PJ. Fetal umbilical artery flow velocity waveforms and placental resistance: pathological correlation. Br J Obstet Gynaecol. 1985;92:31-8.

25. Arduini D, Rizzo G. Normal values of Pulsatility Index from fetal vessels: a crosssectional study on 1556 healthy fetuses. J Perinat Med. 1990;18:165-72.

26. Acharya G, Wilsgaard T, Berntsen GK, Maltau JM, Kiserud T. Reference ranges for serial measurements of umbilical artery Doppler indices in the second half of pregnancy. Am J Obstet Gynecol. 2005;192:937-44.

27. Prefumo F, Venturini PL, De Biasio P. Effect of fetal gender on first-trimester ductus venosus blood flow. Ultrasound Obstet Gynecol. 2003;22:268-70.

28. Teixeira LS, Leite J, Castro Viegas MJ, Faria MM, Pires MC, Teixeira HC, Teixeira RC, Pettersen $\mathrm{H}$. Non-influence of fetal gender on ductus venosus Doppler flow in the first trimester. Ultrasound Obstet Gynecol. 2008;32:12-4.

29. Clur SA, Oude Rengerink K, Mol BW, Ottenkamp J, Bilardo CM. Is fetal cardiac function gender dependent? Prenat Diagn. 2011;31:536-42.

30. Prior T, Wild M, Mullins E, Bennett P, Kumar S. Sex specific differences in fetal middle cerebral artery and umbilical venous Doppler. PLoS One. 2013;8:e56933.

31. Schalekamp-Timmermans S, Cornette J, Hofman A, Helbing WA, Jaddoe WW Steegers EA, Verburg BO. In utero origin of sex-related differences in future cardiovascular disease. Biol Sex Differ. 2016;7:55.

32. Widnes C, Flo K, Acharya G. Exploring sexual dimorphism in placental circulation at 22-24 weeks of gestation: a cross-sectional observational study. Placenta. 2017:49:16-22.

33. Hadlock FP, Harrist RB, Sharman RS, Deter RL, Park SK. Estimation of fetal weight with the use of head, body, and femur measurements-a prospective study. Am J Obstet Gynecol. 1985;151:333-7.
34. Safety Group of the British Medical Ultrasound Society. Guidelines for the safe use of diagnostic ultrasound equipment. Ultrasound. 2010;18:52-9.

35. Gosling RG, Dunbar G, King DH, Newman DL, Side CD, Woodcock JP, Fitzgerald DE, Keates JS, MacMillan D. The quantitative analysis of occlusive peripheral arterial disease by a non-intrusive ultrasonic technique. Angiology. 1971;22:52-5.

36. Pourcelot L. Ultrasonic Doppler velocimetry. Clinical applications of Doppler instruments. COLLINSERM, Paris. 1974;34:213-40.

37. Stuart B, Drumm J, FitzGerald DE, Duignan NM. Fetal blood velocity waveforms in normal pregnancy. BJOG Int J Obstet Gynaecol. 1980;87:780-5.

38. Acharya G, Wilsgaard T, Berntsen GK, Maltau JM, Kiserud T. Doppler-derived umbilical artery absolute velocities and their relationship to fetoplacental volume blood flow: a longitudinal study. Ultrasound Obstet Gynecol. 2005;25:444-53.

39. Gudmundsson S, Fairlie F, Lingman G, Marsal K. Recording of blood flow velocity waveforms in the uteroplacental and umbilical circulation: reproducibility study and comparison of pulsed and continuous wave Doppler ultrasonography. J Clin Ultrasound. 1990;18:97-101.

40. Royston P, Altman DG. Regression using fractional polynomials of continuous covariates: parsimonious parametric modelling. Appl Statist. 1994:43:429-67.

41. Melamed N, Meizner I, Mashiach R, Wiznitzer A, Glezerman M, Yogev Y. Fetal sex and intrauterine growth patterns. J Ultrasound Med. 2013:32:35-43.

42. Kiserud T, Piaggio G, Carroli G, Widmer M, Carvalho J, Neerup Jensen L, Giordano D, Cecatti JG, Abdel Aleem H, Talegawkar SA, et al. The World Health Organization fetal growth charts: a multinational longitudinal study of ultrasound biometric measurements and estimated fetal weight. PLoS Med. 2017;14:e1002220.

43. Owen P, Murphy J, Farrell T. Is there a relationship between estimated fetal weight and umbilical artery Doppler impedance indices? Ultrasound Obstet Gynecol. 2003;22:157-9.

44. Stark MJ, Clifton VL, Wright IM. Sex-specific differences in peripheral microvascular blood flow in preterm infants. Pediatr Res. 2008;63:415-9.

45. Sykes SD, Pringle KG, Zhou A, Dekker GA, Roberts CT, Lumbers ER. The balance between human maternal plasma angiotensin II and angiotensin 1-7 levels in early gestation pregnancy is influenced by fetal sex. J ReninAngiotensin-Aldosterone Syst. 2014;15:523-31.

46. Thompson RS, Trudinger BJ. Doppler waveform pulsatility index and resistance, pressure and flow in the umbilical placental circulation: an investigation using a mathematical model. Ultrasound Med Biol. 1990;16:449-58.

47. Adamson SL. Arterial pressure, vascular input impedance, and resistance as determinants of pulsatile blood flow in the umbilical artery. Eur J Obstet Gynecol Reprod Biol. 1999;84:119-25.

48. Adamson SL, Langille BL. Factors determining aortic and umbilical blood flow pulsatility in fetal sheep. Ultrasound Med Biol. 1992;18:255-66.

49. Saunders HM, Burns PN, Needleman L, Liu JB, Boston R, Wortman JA, Chan L. Hemodynamic factors affecting uterine artery Doppler waveform pulsatility in sheep. J Ultrasound Med. 1998;17:357-68.

50. Adamson SL, Morrow RJ, Bascom PA, Mo LY, Ritchie JW. Effect of placental resistance, arterial diameter, and blood pressure on the uterine arterial velocity waveform: a computer modeling approach. Ultrasound Med Biol. 1989;15:437-42.

51. Morrow RJ, Bull SB, Adamson SL. Experimentally induced changes in heart rate alter umbilicoplacental hemodynamics in fetal sheep. Ultrasound Med Biol. 1993;19:309-18.

52. Amorim-Costa C, Cruz J, Ayres-de-Campos D, Bernardes J. Gender-specific reference charts for cardiotocographic parameters throughout normal pregnancy: a retrospective cross-sectional study of 9701 fetuses. Eur J Obstet Gynecol Reprod Biol. 2016:199:102-7.

53. Bernardes J, Goncalves H, Ayres-de-Campos D, Rocha AP. Linear and complex heart rate dynamics vary with sex in relation to fetal behavioural states. Early Hum Dev. 2008;84:433-9.

54. Kim KN, Park YS, Hoh JK. Sex-related differences in the development of fetal heart rate dynamics. Early Hum Dev. 2016;93:47-55.

55. Edwards A, Megens A, Peek M, Wallace EM. Sexual origins of placental dysfunction. Lancet. 2000;355:203-4.

56. Murji A, Proctor LK, Paterson AD, Chitayat D, Weksberg R, Kingdom J. Male sex bias in placental dysfunction. Am J Med Genet A. 2012:158a:779-83.

57. Kiserud T, Ebbing C, Kessler J, Rasmussen S. Fetal cardiac output, distribution to the placenta and impact of placental compromise. Ultrasound Obstet Gynecol. 2006;28:126-36. 